\title{
A STUDY OF EPITHELIAL REGENERATION IN THE LIVING EYE
}

BY

\author{
IDA MANN
}

OXFOR D

THERE is general a greement that epithelium heals by migration and multiplication of epithelial cells (Arey, 1936). Migration from the edge of the denuded area occurs first and is followed later by mitosis at some distance from the defect so that the loss is made good by pre-existent cells which move actively across to cover the raw zone, these cells themselves being replaced by cell division behind their orginal positions and not over the wound area. Both events have been demonstrated by observers on microscopic preparations of dead tissue, but it is our object to call attention to an experimental and (in suitable cases) clinical method whereby the process can be watched in action in the living eye.

The first observations similar to these*, were made by Leber (1891), who appears to have been uncertain about the cause of the migration for he was not always able to convince himself of the previous existence of a defect in the epithelium, though he suspected it and in every case a minute defect must have been present as the experiments involved injections into the anterior chamber. An account of Leber's experiments was given by von Hippel in 1928 in which he discussed the phenomenon of epithelial migration in relation to inflammation. 'Ranvier also (1898) dealt with the subject in his work on healing of corneal and conjunctival wounds. He pointed out that in clean wounds of the corneal epithelium only the healing occurs by sliding from both edges of the wound and is complete in 24 hours. Neither plastic lymph, fibrin nor mitoses are required in the healing of small wounds of the cornea. He states that the process does not appear to hold good for conjunctival wounds in which a reaction of the underlying cells is also involved. It would appear probable from the experiments about to be described that if the conjunctival epithelium alone is removed and the sub-epithelial tissue not injured the process of healing by sliding is the same as in the cornea. Werner (1902) and Marchand (1924) have also described the sliding process. H. W. Florey and $\mathrm{H}$. E. Harding have observed the same phenomenon in experimental ulcers of the duodenum.

The most convincing microscopic demonstration of the process has been given by V. B. Wigglesworth in his paper on "Wound

\footnotetext{
- The process was first observed in 1941 when studying the effects of mustard gas.
} 
Healing in an Insect" from which Fig. 1 has been taken. $\mathrm{He}$ used a blood-sucking bug (Rhodnius prolixus hemiptera) in which, once growth is complete, no further mitoses occur in the simple layered epithelium. His preparations show the stages of activation of the cells along the edges of the wound, followed by migration to the site of injury over which they spread. This leads to a thinning of the cells in the zone further out where mitoses then occur to bring the number of cells back to normal. Fig. 1 shows the covering of the defect by thinning, and sliding and also the

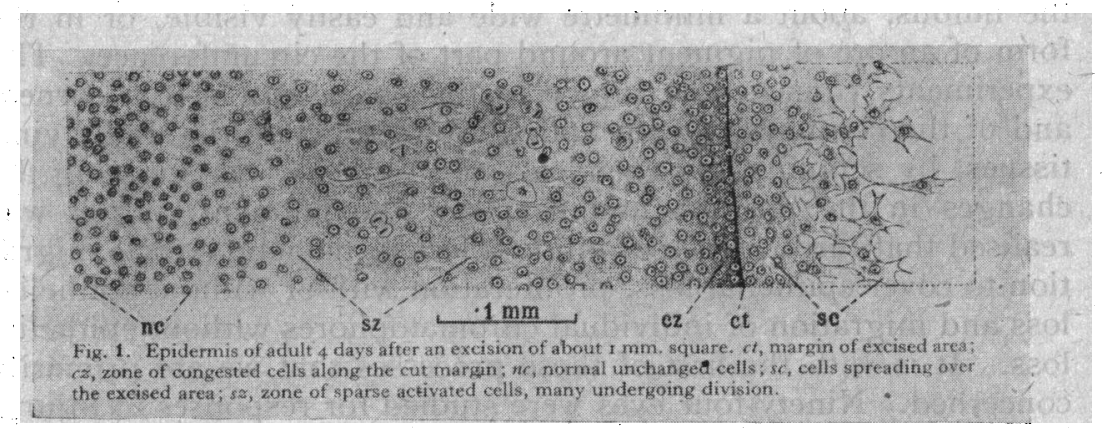

FIG. 1 .

Reproduced by kind permission of Professor Wigglesworth from the Journal of Experimental Biology, 1937, Vol. XIV., p. 368. This plate shows the arrangement of cells in the healing insect epidermis. Epider mis of adult 4 days after an excision of about $1 \mathrm{~mm}$. square. $c t$. margin of excised area; $c z$, zone of congested cells along the cut margin; nc, normal urchanged cells; sc, cells spreading over the excised area; $s z$, zone of sparse activated cells, many undergoing division.

zone of mitoses. He states that in this animal the migration begins in 6 hours and is usually complete in 2-3 days. The mitoses in the adjacent zone increase to a peak in 6-7 days. He considers that the substance which activates the sliding cells is a product of the autolysis of the injured cells and is neither tissue nor species specific. It is destroyed by heat so that the healing of a burn may be accompanied by mitoses at the spreading edge itself and not a migration.

The experiments which we are about to describe are applicable to any species which shows pigment in the basal cells of the conjunctival or corneal epithelium, since the presence of the pigment allows one to recognise the particular cells involved and to follow their migration from day to day, in response to various types and sites of injury. The movement of the pigment can be seen in many' cases with the naked eye and in all cases with a corneal loupe. Slit-lamp examinations were also made, but are not necessary for the observation of the phenomenon. The presence of the 
initial loss of epithelium was assured by the use of fluorescein and in no case was a slide observed apart from an epithelial defect. The observations were made on rabbits and were confirmed clinically in man on Africans.

Experiments.-A series of 120 eyes of 82 rabbits of crossed Dutch strain (black and white) with some pure black and some wild colour with brown eyes were used. These rabbits were all suitable because they had pigment in the basal cells of the limbal epithelium, either in the form of a complete brown ring around the limbus, about a millimetre wide and easily visible, or in the form of an arc of pigment around part of the circumference. The experiments consisted of injury to the epithelium of the cornea, and of the conjunctiva with and without injury to the underlying tissues, by scraping, by chemicals and by heat. In studying the changes in the position and shape of the pigment ring it was realised that three processes might affect it, namely, simple migration to cover epithelial loss, proliferation with or without epithelial loss and migration of individual chromatophores without epithelial loss. It is with the first of these processes that we are primarily concerned. Ninety-four eyes were studied for responses to injury. Twenty eyes showing pigment proliferation as distinct from migration were studied and will be briefly described for contrast with the first group, and to make the picture of migration in response to injury complete, six eyes were observed in which migration without injury had been induced by dietary deficiency. These three types are quite distinct and can be readily distinguished so that in the series of 94 eyes there is no doubt that the movement of the pigment ring occurred as a result of the loss of epithelium.

The first series of experiments is designed to demonstrate the movement in its simplest form in response to simple superficial injury: If a rabbit's cornea is abraded with a Tuke's knife or other suitable instrument over a narrow area $2.5 \mathrm{~mm}$. from the pigment at the limbus and parallel with it, taking care not to injure the underlying substantia propria, the pigment ring adjacent to the injury will show an irregularity, apparent in less than 20 hours. This (Fig. 2A and B) irregularity affects the inner but not the outer edge of the pigment band and gradually increases until an even fringe has almost reached the denuded area. It does not pass across this, as it meets the slide from the opposite side which in the absence of pigment cannot be seen in life, but can be inferred from the position of the pigment and from the loss of staining with fluorescein. The margins of the slide on both sides show with the slit-lamp a faint epithelial bedewing which disappears when the two edges have met. The pigment in the slide remains stationary and visible macroscopically (Fig. 2a) for some time, and 
very gradually becomes sparser, scattered grains remaining visible for many months, but being finally lost as the cells containingthem are eventually shed. The original limbal pigment ring persists, though it may show a sparse area for some time.

If, after such a.pigment fringe has been produced and has healed, the cornea is further injured by scraping off the epithelium

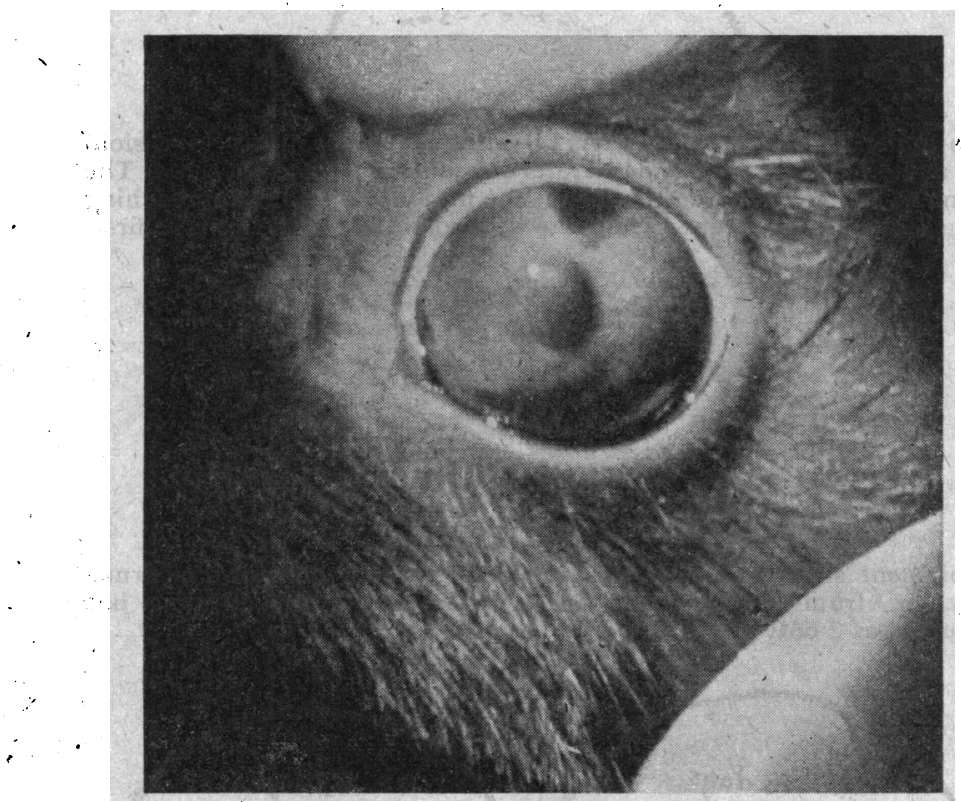

FIG. 2A.

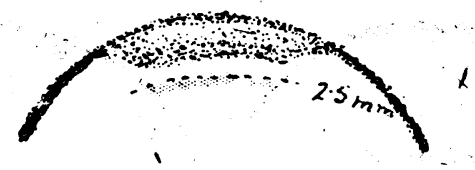

FiG. 2B

(a) Photograph of rabbit's eye with triangular pigment slide above, easily visible macrcscopically.

(b) The upper limbus of a rabbit's cornea. The shaded portion shows the area over which the epithelium had been scraped off the previous day. It was $2.5 \mathrm{~mm}$. from the limbus. The pigment ring (dotted area) is sliding towards it as a tringe.

as before, but over a similar area $2.5 \mathrm{~mm}$. nearer to the centre of the cornea, a further slide can be produced, the original pigment fringe crossing over the site of the original injury and approaching, but not necessarily reaching, the centre of the 
new injury (Fig. 3). ' In this way a slide can be made to approach -the centre of the cornea in successive stages, but if the initial injury is made $5 \mathrm{~mm}$. from the limbus, then no movement of the

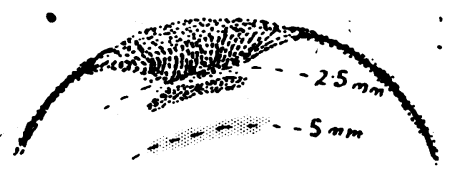

FIG. 3 .

The upper limbus (seen in Fig. 2a) after a further injury (abrasion) $2.5 \mathrm{~mm}$. from the first and therefore $5 \mathrm{~mm}$. from the limbus. The pigment has now crossed the site of the first injury and is approaching the second: Eight days from the second and 11 days from the first.

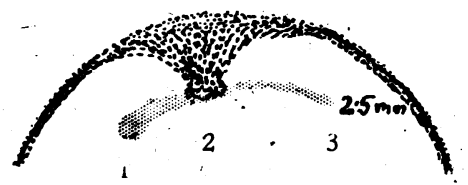

FIg. 4.

A pigment slide towards a portion (central) of a burn of the cornea $2.5 \mathrm{~mm}$. from the limbus." Two days after injury. Sliding has occurred at 2 only.
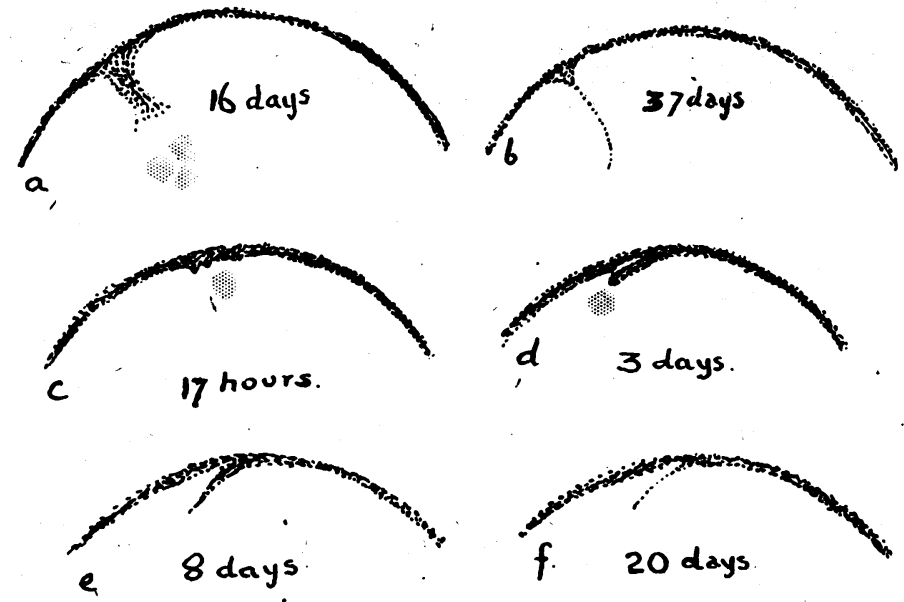

FIG. 5. Diagrams showing a tongue-like slide.

(a) A narrow fringe running towards a small lesion, the previous position of which is shown as three shaded spots. Sixteen days. (b) The same slide at 37 days.

$(c, d, e, f)$ Four successive stages in the formation of a linear slide in response to a small round abrasion $2.5 \mathrm{~mm}$. from the limbus. 
- pigment ring will take place. In this case there is sufficient unpigmented epithelium between the wound and the limbus to cover the denuded area. This gives us some idea of the area of spread of the activating substance from a simple removal of epithelium. It seems to be more than 2.5 and less than $5 \mathrm{~mm}$. from the edges of the wound in all directions. If instead of removing the epithelium by scraping, the cornea is burned with a hot wire then the amount of sliding produced is very much less, the movement of the pigment being very irregular and not occurring all along the burned area. It is probable that migration occurs only towards the less severe parts of the burn if W.igglesworth's contention that heat destroys the activation is correct. Fig. 4 shows three small burns, the first and third being made with a hotter wire than the second, towards which alone migration has occurred, though all three have healed, the first and third presumably by mitotic division from their edges.

If instead of abrading an area parallel with the limbus a small round lesion is made (at $2.5 \mathrm{~mm}$. from the edge of the pigment) then instead of a fringe, a linear slide will be produced. At 24 hours there will be a slight irregularity and at three days a narrow tongue of pigment will detach itself and approach the wound, becoming longer and thinner as this heals (Fig. 5). If the conjuntival epithelium is removed without injury to sub-conjunctival tissues then a reversed slide will be produced by the corneal epithelium migrating outwards to make up the loss, (Fig. 6). Sometimes only fringing occurs, sometimes the whole limbal band migrates, as in Fig. 6, in which the destruction of the conjunctival epithelium was produced by a minute application of mustard gas and not by scraping.

If the pigment ring itself is scraped off there is very little tendency for regeneration by migration of the two ends towards each other. The sliding appears to occur at an angle to the limbus and not along it. The abrasion heals in three days or less, but pigment does not appear in any quantity in the gap until the eighth day, when scattered grains are visible which only very slowly in the course of months spread in the damaged area, and never completely reconstitute it.

The second series of experiments deals with the form of slide produced by damage to larger areas of the cornea. In these experiments the epithelium was destroyed by the use of chemicals. In some cases the epithelium alone was damaged and in others the corneal corpuscles in the underlying area were killed to varying depths. In all cases, however, the behaviour of the limbal pigment ring was determined by the area of destruction of the corneal epithelium and not by the condition of the substantia propria nor 

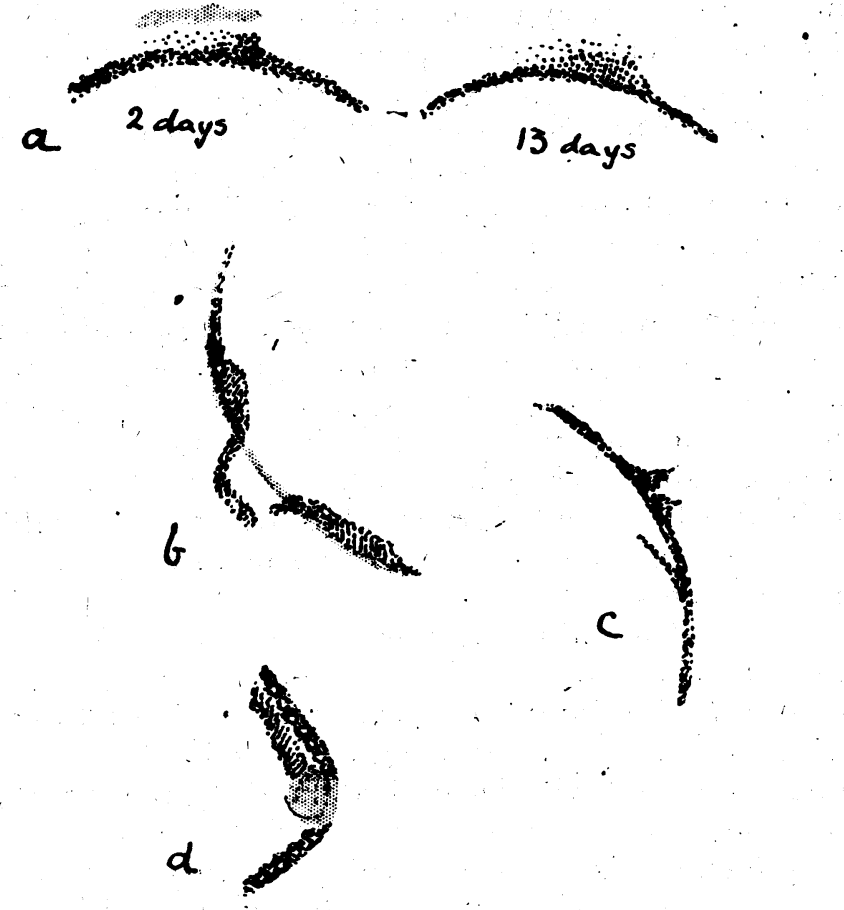

Fig. 6.

(a)' Two stages in a reversed pigment fringe produced by an abrasion of the conjunctival epithelium. The shaded portion shows the position of the lesion.

(b) A slide of the whole width of the pigment ring on to the conjuuctiva following an in jury with mustard gas.

(c) Fringes on to cornea and conjunctiva from very small lesions on either side of the limbus.

(d) A pigment fringe on the 3rd lid of a rabbit produced by a lesion with mustard gas in the shaded area.

by the nature of the chemical used. Even in cases in which the corneal corpsucles were destroyed and the substantia propria rendered oedematous, the process of healing occurred by migration in the same way, and during the same, period as when the injury was a simple abrasion. This also held good when the injury was sufficient to produce vascularisation which proceeded subepithelially after healing. Although changes in the epithelium covering such an injured substantia propria might occur later (secondary oedema or complete breakdown) in most cases healing of the superficial loss was complete (as evidenced by disappearance of staining with fluorescine) in four days, while the pathological process continued its course deep to the epithelium for days or sometimes weeks 
afterwards. This finding is in agreement with the recognised conception of the epithelium being as it were parasitic on the substantia propria; it is probable that it receives some at least of its nutriment from its superficial surface, i.e., via the lid and lacrimal secretions and is not dependent on the state of the underlying substantia propria for its existence (Pirie).

The chemicals used to destroy the epithelium included some, such as acetone, which destroy the epithelium alone, and others, for example formalin, which penetrate and temporarily alter the whole thickness of the cornea. Hydrochloric acid, chloroform, ammonia and mustard gas all produced similar results on the epithelium, though they differed from each other in their deeper action. A variety of chemical warfare agents was tried, but the chemical used appeared to be immaterial so long as the localised epithelial injury resulted. The types of migration would seem to fall into categories based on shape and to be determined by the

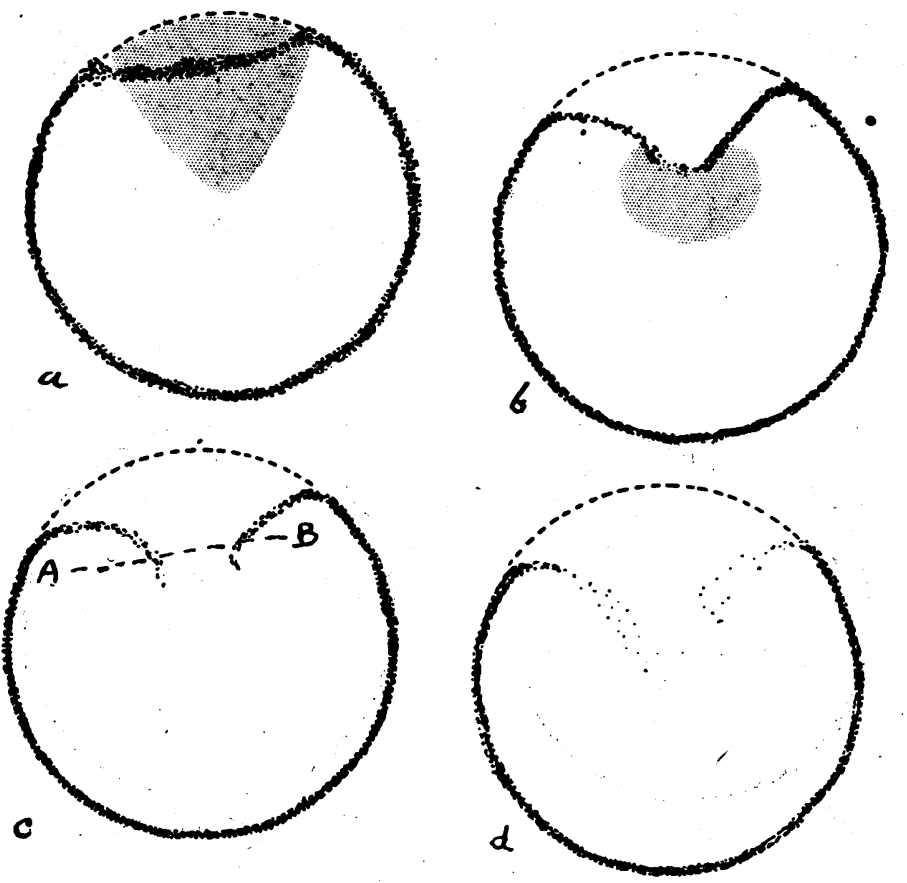

FIG. 7.

Four stages in the healing of a large sectorial lesion (shown shaded in a). The limbal pigment ring slips inwards without a break until the staining has gone at four days. The break occurs $(b \in c)$ at six to seven days and by 14 days $(d)$ the pigment is sparse and widely scattered. The gap in the ring at the limbus is permanent. The line A B in (c) is the line of section in Fig. 9. 
size and site of the initial denuded area as shown by fluorescein staining.

The movement of the pigment-containing cells starts at or before 18 hours. It is most obvious on the 3 rd or 4 th day (average from 93 cases) and reaches its maximum always within a week. After this the pigment grains begin to thin out and appear sparser, gradually disappearing. In some cases of small slides no trace is left after 10 days, but in many cases traces persist for 3 weeks and in some for as long as 4 months. In practically every case the defect was covered (as evidenced by cessation of staining with fluorescein) in 4 days.

We can consider first the effect of a sectorial lesion (Fig. 7) which produces a displacement inwards of the whole segment of the limbal pigment ring. This may break in its centre and the two ends curve in towards the centre of the cornea. The pigment grains become sparser and gradually disappear. In the rabbit illustrated in Fig. 7 the inward displacement was marked on the 4th day, by which time there was no staining. The break occurred on the 7th day. By three weeks there was very little pigment left
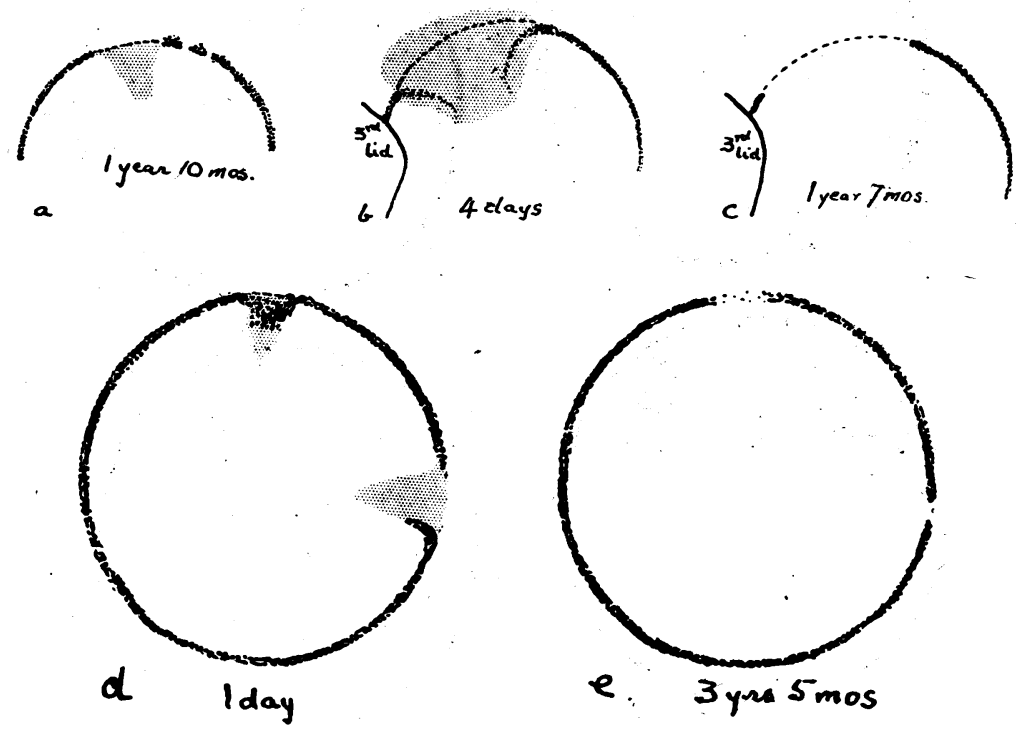

FIG. 8 .

Three cases of break in the limbal ring without regeneration, (a) 1 year 10 months after chemical injury in shaded area, $(b \& c)$ two stages of a limbal lesion (four days and 1 year 7 months), $(d \& \dot{e})$ two stages of two minimal limbal lesions in the same eye (1 day and 3 years 5 months) showing that only very slight regeneration occurs even in
small breaks. 
and in a month it had disappeared. The gap in the limbal pigment never completely regenerates when the whole width of the ring has migrated. Irregularities, thin areas and actual gaps persist and have been observed for $3 \frac{1}{2}$ years, even after relatively small breaks. The pigment which is displaced, however, disappears entirely and there is no attempt at persistence in the new position (Fig. 8). When fringing only has occurred, as pointed out in the first series of experiments, the migrated pigment disappears, but the ring from which it came persists though it is often a little sparser opposite the slide for some time. The permanent disappearance of the pigment after a complete slide is curious, since if the basal cells have taken part one would expect them to continue to produce pigment in their new situation and if they have not, one would expect the ring to regenerate from them in the original position. Fig. 9 appears to show migration of the whole thickness of the epithelium including the basal cells. It represents a section through a pigment slide.

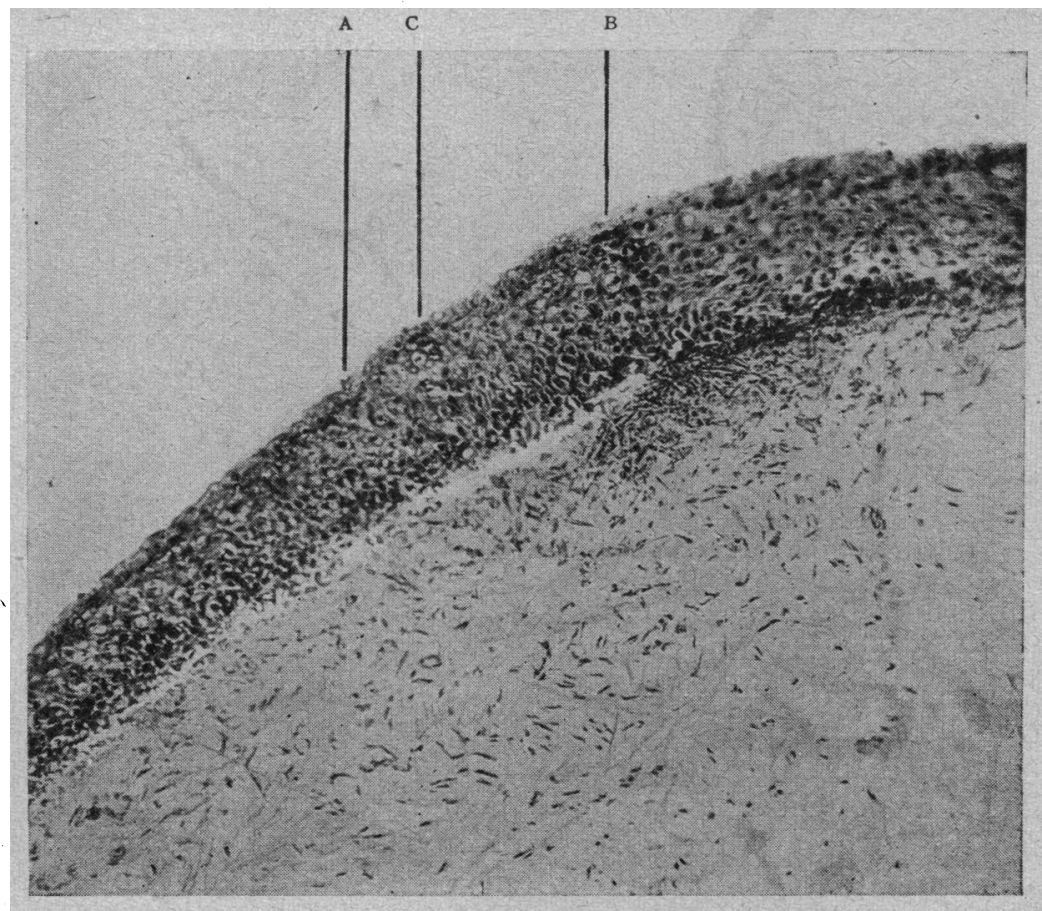

FIG. 9.

Microphotograph $\times 100$ of the corneal epithelium in the pigment slide see in Fig. 7c, showing the pigment and a polymorphonuclear reaction in the underlying substantia propria.

$(a \& b)$ Pigment in the epithelial cells.

(c) Mucus cells derived from the conjunctiva by sliding. 
If the lesion is not quite so extensive then the actual break may not occur, the pigment line being merely displaced on to the cornea and gradually fading away, i.e., disappearing from, stage 1 of Fig. 7.

If the lesion is very extensive the most widespread and bizarre slides may be produced. Fig. 10 shows the maximal slide produced. The epithelium of practically the whole cornea except the

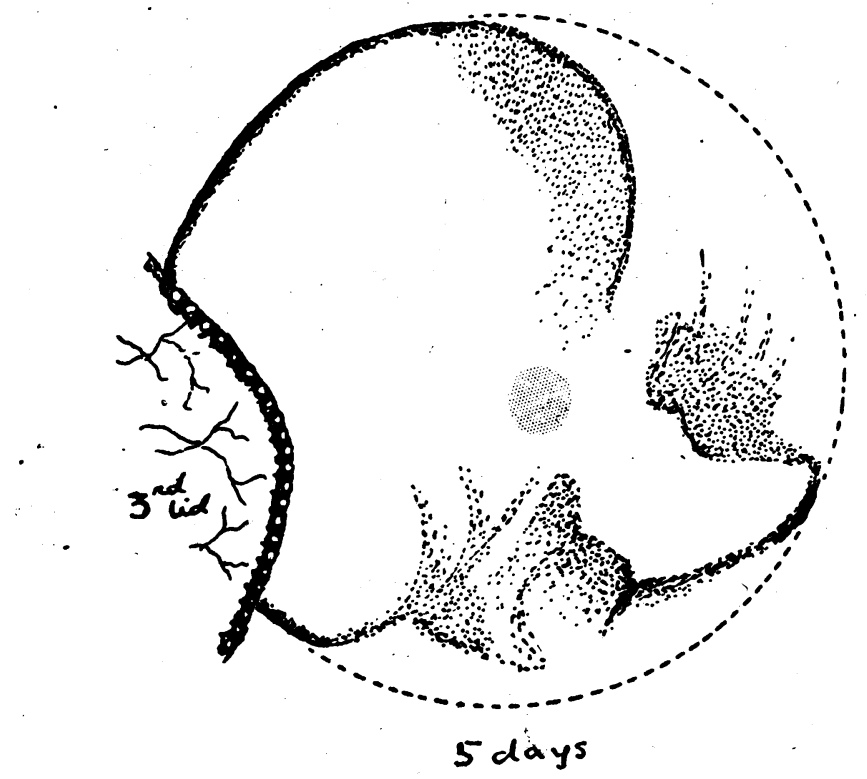

FIG. 10.

A very extensive pigment slide five days after almost complete destruction of the corneal epithelium.

limbus had been destroyed.' Fig. 11 shows two other large pigment slides of varying pattern.

These experiments emphasize this method of corneal healing and show how the relatively large areas of destruction are made good in so short a time. This is in line with the common clinical experience of the rapid healing of corneal abrasions in man, but owing to the absence of any landmark it is impossible to be sure that the process is one of migration and not of cell division. If, however, the eyes of Nigerians, who possess a pigment ring even wider and more striking than that of a rabbit, are examined after small injuries such as the removal of a corneal. foreign body, a similar demonstration of sliding can be seen. Fig. 12 shows two pigment slides (a fringe and a linear migration) from 
the eyes of two Nigerians. One (a) only vaguely remembered the injury so that it must have been some time before, the other $(b)$ complained that a spark or small foreign body in a blacksmith's shop had gone into his eye a few days before.

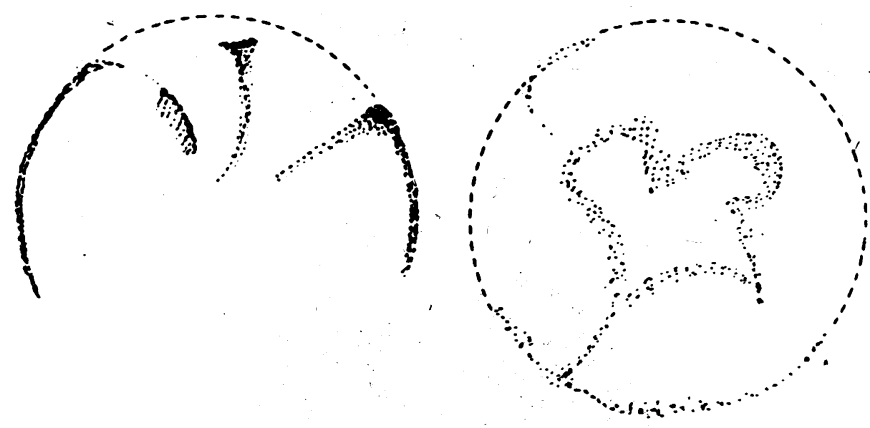

FIG. 11

Two large pigment slides.

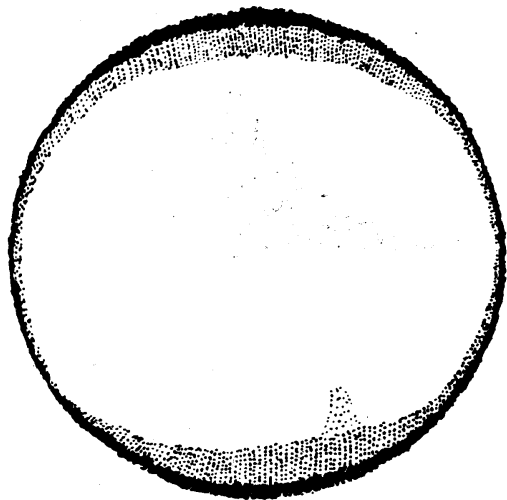

a

The third series of experiments deals with the production of pig-
ent proliferation in response to chemical injury and its differen-
gment proliferation in the rabbit appears to be by injuring the
e with any of the arsenical war gasses. The injury should be

The third series of experiments deals with the production of pig-
ment proliferation in response to chemical injury and its differen-
tiation from simple migration. The simplest way of producing
pigment proliferation in the rabbit appears to be by injuring the
eye with any of the arsenical war gasses. The injury should be

The third series of experiments deals with the production of pig-
ment proliferation in response to chemical injury and its differen-
tiation from simple migration. The simplest way of producing
pigment proliferation in the rabbit appears to be by injuring the
eye with any of the arsenical war gasses. The injury should be

The third series of experiments deals with the production of pig-
ment proliferation in response to chemical injury and its differen-
tiation from simple migration. The simplest way of producing
pigment proliferation in the rabbit appears to be by injuring the
eye with any of the arsenical war gasses. The injury should be

The third series of experiments deals with the production of pig-
ment proliferation in response to chemical injury and its differen-
tiation from simple migration. The simplest way of producing
pigment proliferation in the rabbit appears to be by injuring the
eye with any of the arsenical war gasses. The injury should be

The eyes of two Nigerians after corneal foreign bodies. In Nigerians the pigment is present on the cornea itself as well as the limbus proper. It forms a ring wider above and below, encircling the cornea. 
severe enough to produce a dense vascularising keratitis, but not sufficient to cause perforation and collapse of the globe. The matter is only of interest here in showing the distinction between such increase and misplacement of pigment and that due to the epithelial migration described above. The pigment proliferation did not occur until after the healing of the epithelium, so that it was never apparent within the first 4 days, as the pigment slide always was. The prolifieration was produced in 20 eyes, the earliest time being 30 days after the original injury and the longest

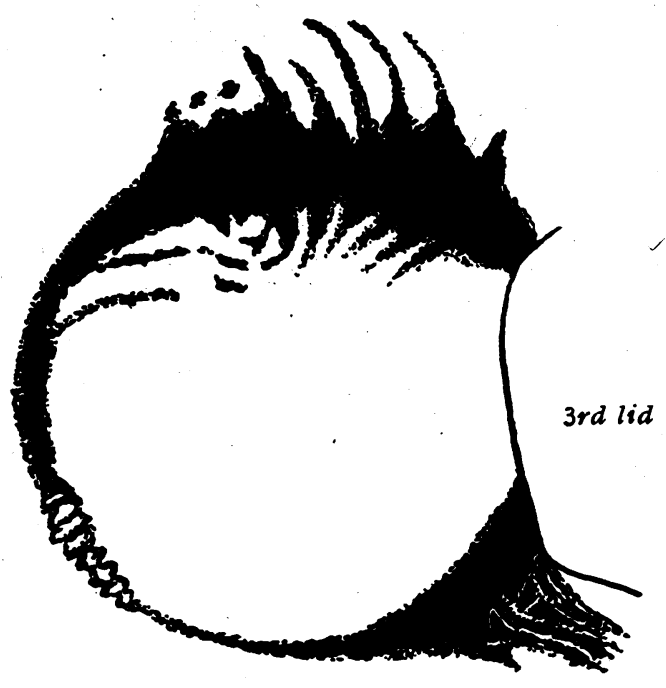

FIG. 13.

Dense pigment proliferation in a rabbit's eye two years after an injury with Lewisite.

nine weeks, though in this case it may have started somewhat earlier. The overgrowth of pigment was progressive and continued to increase for months (in one case for two years). The appearance was distinctive, the pigment being in dense masses arising from the limbus and spreading both on to the cornea and on to the conjunctiva (Fig. 13). There had not necessarily been an original pigment slide. The figure shows the very distinctive appearance which is not likely to be mistaken for a simple epithelial slide.

The fourth series of experiments exemplifies an already known phenomenon of pigment migration from the pigment ring on to 
the conjunctiva in cases of vitamin $A$ deficiency. This migration gives rise in man to the diagnostic smoky conjunctiva of A deficiency in pigmented races. It can be produced experimentally in rabbits and serves as an example of pigment migration without epithelial loss. It is not in the nature of a generalised slide, but

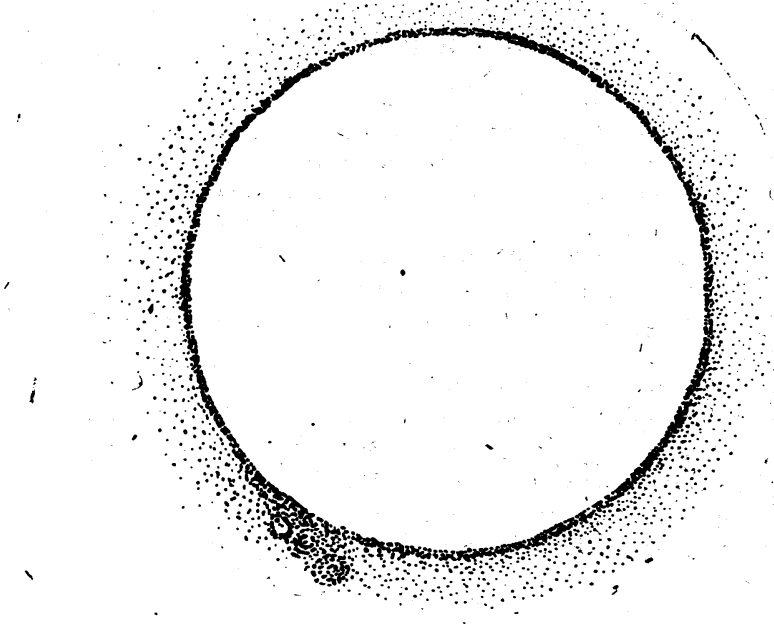

Fig. 14.

Diffuse migration of pigment from the limbus to the conjunctiva without antecedent injury in vitamin $A$ deficiency in a rabbit.

suggests a wandering of chromatophorés one by one from the pigment ring outwards towards the fornices. Fig. 14 shows a diagram of the limbus of a vitamin A deficient rabbit. The appearance is in no way to be confused with either proliferation or sliding and there has been no preliminary epithelial loss.

\section{Summary}

1. The sliding or migration of healing epithelium has been demonstrated in the living eyes of rabbits possessing a pigment ring in the conjunctiva at the limbus, and also in Nigerians who show the same condition.

2. The slide can be produced by simple trauma or by chemical injury. It is not so easily produced by heat.

3. The shape of the slide is determined by the shape and position of the epithelial loss and can occur on to the conjunctiva or the cornea equally. 
4. The rate and shape of the slide do not appear to be influenced by the nature of the injury nor the condition of the underlying substantia propria.

5. The sliding of a pigmented limbus after trauma can be distinguished from pigment proliferation after chemical stimuli and from pigment migration without epithelial loss in -vitamin A deficiency.

\section{BIBLIOGRAPHY}

AREY.-Phys. Review, Vol. XVI, p. 327, 1936.

AREY and Covode.-Anat Rec, Vol. LXXXVI, p. 75, 1943:

Florey, H. W. and HARDING, H. E.-Jl. Path. aud Bact., Vol. XL, p. 211; 1935. von HiPPEL.-Handbuch der spec. Path. Anat. und Hist., Bd., Vol. XI, i, p. 336 1928.

LEBER.-Die Entstehung der Entzundung. 500. 1891.

LOEB.-Arch. f. Entwick.-Mech. d. Org., V̇ol: VI, p. 297, 1898.

Mann, I. and Pullinger, B. D.-Proc. Roy. Soc. Med., Vol. XXXV, p. 229, No. 3, January, 1942.

- Jl. Path. and Bact., Vol. LV, p. 151, 1943.

MARCHAND.-Handbuch der allgemeinen Path., Bd. 4, Abt. 1, Leipzig, 1924.

PIRIE, A. and Philpot, F. J.-Biochem. Jl., Vol. XXVII, No. 2, p. 250, July, 1943

RANVIER.-Arch.d'Anat. Micros., ii, p. 77, 1898.

WERNER.-Beitrag. z. Klin. Chir., Vol. XXXIV, p. 1, 1902.

Wigglesworth, V. B.-Jl. Exper. Biol., Vol. XIV, p. 364, 1937.

\section{ATAXIC NYSTAGMUS: A PATHOGNOMONIC SIGN IN DISSEMINATED SCLEROSIS}

BY

\section{WILFRED HARRIS}

LONDON

For many years I have observed this peculiar form of nystagmus, and have demonstrated it to numbers of students and to my clinical assistants as being diagnostic of disseminated sclerosis. In my experience it is not met with in any other condition. On June 28, 1943, I showed a case illustrating this condition at the clinical meeting of the Medical Society of London, held in the wards of St. Mary's Hospital. As this aroused a certain amount of interest, and no-one who saw the case appeared to have observed this form of nystagmus previously, I feel that a description of its peculiarity should be put on record.

When the eyes are turned laterally, the conjugate action appears weak, so that the inner eye, e.g., the right eye when looking 